\title{
HIGHLIGHTS
}

DIABETES

\section{Long-term metformin exposure linked with peripheral neuropathy in patients with type 2 diabetes mellitus}

Cumulative metformin exposure is strongly associated with the severity of peripheral neuropathy in patients with type 2 diabetes mellitus (T2DM), show Daryl Wile and Cory Toth at the University of Calgary.

Peripheral neuropathy affects up to $50 \%$ of patients with T2DM. Vitamin $\mathrm{B}_{12}$ deficiency is also common in patients with T2DM and is an independent cause of peripheral neuropathy and other nervous system diseases. Metformin therapy has been associated with vitamin $B_{12}$ deficiency in patients with T2DM, but, Wile explains, "its effects on the peripheral neuropathy of T2DM have not been systematically studied."

In a prospective case-control study of patients with T2DM and symptomatic peripheral neuropathy, the investigators compared 59 patients who had been treated with metformin for more than 6 months with 63 patients who had never taken metformin. "We assessed the clinical status of the patients based on the validated Toronto Clinical Scoring System (TCSS) and Neuropathy Impairment Score (NIS) scales," explains Wile. High scores indicate more severe neuropathy. The median scores for the metformin-treated group were significantly higher than those for the group who had not received metformin and were positively correlated with cumulative metformin dose.

"We also wanted to see if we could find biochemical correlates to the clinically more severe neuropathy we expected to find," adds Wile, "so we looked for evidence of $B_{12}$ deficiency in our metformin-exposed patients." Patients treated with metformin had significantly lower median serum concentrations of vitamin $B_{12}$ than those not exposed to metformin, and higher median fasting serum concentrations of homocysteine and methylmalonic acid, two metabolites that are sensitive indicators of vitamin $B_{12}$ deficiency. Cumulative metformin doses correlated inversely with serum vitamin $B_{12}$ levels and positively with serum homocysteine and methylmalonic acid levels.

"Our aim is to raise awareness of an identifiable and treatable potential contributor to peripheral neuropathy severity," points out Wile. The researchers recommend screening for vitamin $B_{12}$ deficiency in patients with T2DM and peripheral neuropathy at the start of metformin therapy and at intervals of 1-2 years thereafter.

Joana Osório

Original article Wile, D. J. \& Toth, C. Association of metformin, elevated homocysteine, and methylmalonic acid levels and clinically worsened diabetic peripheral neuropathy. Diabetes Care 33, 156-161 (2010) 